\title{
Using DEA and AHP for Multiplicative Aggregation of Hierarchical Indicators
}

\author{
Mohammad Sadegh Pakkar \\ Faculty of Management, Laurentian University, Sudbury, Canada \\ Email: $\underline{\text { ms pakkar@laurentian.ca }}$
}

Received 23 June 2015; accepted 21 July 2015; published 24 July 2015

Copyright (C) 2015 by author and Scientific Research Publishing Inc.

This work is licensed under the Creative Commons Attribution International License (CC BY).

http://creativecommons.org/licenses/by/4.0/

(c) (i) Open Access

\begin{abstract}
The author [Pakkar, M.S. (2014) Using Data Envelopment Analysis and Analytic Hierarchy Process to Construct Composite Indicators. Journal of Applied Operational Research, 6(3), 174-187.] recently proposed a multiplicative approach using Data Envelopment Analysis (DEA) and Analytic Hierarchy Process (AHP) to reflect the priority weights of indicators in constructing composite indicators (CIs). Nonetheless, this approach is limited to the situations with a single level hierarchy which might not satisfy the needs of a multiple level hierarchy. Therefore, the current paper extends this approach to the situations in which the indicators of similar characteristics can be grouped into sub-categories and further linked into categories to form a three-level hierarchical structure. An illustrative example of road safety performance for a set of European countries highlights the usefulness of the proposed "extended approach".
\end{abstract}

\section{Keywords}

Data Envelopment Analysis, Analytic Hierarchy Process, Composite Indicators, Multiplicative Aggregation, Hierarchical Structures

\section{Introduction}

A composite indicator (CI) is a mathematical tool to aggregate a set of multidimensional indicators in order to produce a single measure of performance. In a recent paper, Pakkar [1] proposed a multiplicative approach using DEA and AHP to reflect the priority weights of indicators in constructing CIs. This approach can be organized into the following steps:

1) Using a multiplicative DEA based-CI model to compute the composite value of each Decision Making Unit (DMU). The computed composite values are used in the next step.

2) Using a minimax distance model to obtain the optimal weights of indicators for each DMU (minimum composite loss). 
3) Using the minimax distance model bounded by AHP to obtain the priority weights of indicators for each DMU (maximum composite loss).

4) Using a parameter goal programming model to assess the performance of each DMU in terms of its relative closeness to the priority weights of indicators.

In the basic multiplication DEA based-CI models, all indicators are simply treated to be at the same level of hierarchy [2]. Nonetheless, these indicators might also belong to different sub-categories and further be linked to one another constituting a three-level hierarchical structure. To overcome this limitation, we integrate AHP to a three-level DEA-based CI model in a multiplicative context. A three-level DEA based-CI model can reflect the characteristics of the generalized multi-level DEA based-CI model developed by [3].

\section{Methodology}

\subsection{DEA-Based CI Model}

A DEA-based CI model can be formulated similar to a multiplicative DEA model without explicit inputs [2]. In the following, and in line with the more common CI terminology, we will often refer to outputs as "indicators". In order to eliminate the scale differences between all (output) indicators, and moreover, to ensure that all of them are in the same direction of change the normalized counterparts of indicators, using a min-max method, are computed as follows [4]:

$$
\begin{aligned}
& y_{r j}=\left(\frac{\hat{y}_{r j}-\hat{y}_{r(\min )}}{\hat{y}_{r(\max )}-\hat{y}_{r(\min )}}\right)+x, \hat{y}_{r(\max )}=\max \left\{\hat{y}_{r 1}, \hat{y}_{r 2}, \cdots, \hat{y}_{r n}\right\} \text { for desirable indicators, } \\
& y_{r j}=\left(\frac{\hat{y}_{r(\max )}-\hat{y}_{r j}}{\hat{y}_{r(\max )}-\hat{y}_{r(\min )}}\right)+x, \hat{y}_{r(\min )}=\min \left\{\hat{y}_{r 1}, \hat{y}_{r 2}, \cdots, \hat{y}_{r n}\right\} \text { for undesirable indicators. }
\end{aligned}
$$

Here, $y_{r j}$ is the normalized value of (output) indicator $r(r=1,2, \cdots, s)$ for DMU $j(j=1,2, \cdots, n)$. Since in a multiplicative aggregation, the value of each indicator must always be larger than 1 , we add a positive constant $x$ to the normalized values of each indicator. We choose $x$ so that $\left(y_{r(\min )}+x\right)$ turns to 1.01 while $y_{r(\min )}$ is the minimum normalized value of indicator $r$ for all DMUs. Although the model used in this paper does not satisfy the desirable unit invariant property, it is very robust to changes in the measurement units [2]. Therefore, this would only slightly change the composite values without making a significant change in DMUs' rankings. Then a multiplicative optimization model in the construction of a composite indicator can be formulated as $C I_{k}=\max \prod_{r=1}^{s} y_{r k}^{u_{r}}$, subject to $\prod_{r=1}^{s} y_{r j}^{u_{r}} \leq e$ with $u_{r} \geq 0$, where $C I_{k}$ is the composite value of $\mathrm{DMU}_{k}$

$(k=1,2, \cdots, n)$ or the DMU under assessment, $u_{r}$ is the weight of indicator $r(r=1,2, \cdots, s)$ and $e$ is the base of the natural logarithm. Taking logarithms with base $e$, the multiplicative model can be converted to the following log-linear programming model:

$$
\begin{array}{ll}
\operatorname{Max} \quad C \tilde{I}_{k}=\sum_{r=1}^{s} u_{r} \tilde{y}_{r k} \\
\text { s.t. } \\
\sum_{r=1}^{s} u_{r} \tilde{y}_{r j} \leq 1 \quad \forall j, \\
u_{r} \geq 0 \quad \forall r,
\end{array}
$$

where the tilde symbol $(\sim)$ denotes natural logarithms. The combination of (3)-(5) forms a single level DEAbased CI model in a log-linear context that looks like an output-oriented DEA model without explicit inputs. This model is theoretically similar to the log-linear DEA model for efficiency analysis introduced in [5].

\subsection{Three-Level DEA-Based CI Model}

We develop our formulation based on a generalized distance model (for example, see [6]) in such a way that the 
hierarchical structures of indicators, using a weighted-average approach, are taken into consideration [3]. Let $\tilde{y}_{l l^{\prime} j}=\ln \left(y_{l l^{\prime} r j}\right)$ be the value of indicator $r(r=1,2, \cdots, s)$ of sub-category $l^{\prime}\left(l^{\prime}=1,2, \cdots, S^{\prime}\right)$ of category $l(l=1,2, \cdots, S)$ for DMU $j(j=1,2, \cdots, n)$ after normalizing the original data. Let $u_{l l^{\prime} r}$ be the internal weight of indicator $r$ of sub-category $l^{\prime}$ of category $l$ while $\sum_{r=1}^{s} u_{l l^{\prime} r}=1$. Then the value of sub-category $l^{\prime}$ of category $l$ for the DMU $j$ is defined as $\tilde{y}_{l l^{\prime} j}=\sum_{r=1}^{s} u_{l l^{\prime}} \tilde{y}_{l^{\prime} r_{j}}$. Let $p_{l l^{\prime}}$ be the internal weight of sub-category $l^{\prime}$ of category $l$ while $\sum_{l^{\prime}=1}^{S^{\prime}} p_{l l^{\prime}}=1$. Then the value of category $l$ is defined as $\tilde{y}_{l j}=\sum_{l^{\prime}=1}^{S^{\prime}} p_{l l^{\prime}} \tilde{y}_{l l^{\prime} j}$. Let $p_{l}$ be the weight of category $l$. To develop a linear model, the new multiplier of indicator $r$ of sub-category $l^{\prime}$ of category $l$ is defined as: $u_{l l^{\prime} r}^{\prime}=p_{l} p_{l l^{\prime}} u_{l l^{\prime} r}$. Similarly, the new multiplier of sub-category $l^{\prime}$ of category $l$ is defined as: $p_{l l^{\prime}}^{\prime}=p_{l} p_{l l^{\prime}}$. Let $C \tilde{I}_{k}^{*}(k=1,2, \cdots, n)$ be the best attainable composite value for the DMU under assessment, calculated from the one-level DEA-based CI model. We want the composite value $C \tilde{I}_{k}\left(u_{l^{\prime} r}^{\prime}\right)$, calculated from the set of weights $u_{l l^{\prime} r}^{\prime}$ to be closest to $C \tilde{I}_{k}^{*}$. Our definition of closest is that the largest distance is at its minimum. Hence we choose the form of the minimax model: $\min _{u_{u^{\prime} r} r} \max _{k}\left\{C \tilde{I}_{k}^{*}-C \tilde{I}_{k}\left(u_{l^{\prime} r}^{\prime}\right)\right\}$ to minimize a single deviation which is equivalent to the following linear model:

Min $\theta$

s.t.

$$
\begin{array}{ll}
C \tilde{I}_{k}^{*}-\sum_{l=1}^{S} \sum_{l^{\prime}=1}^{S^{\prime}} \sum_{r=1}^{s} u_{l l^{\prime}}^{\prime} \tilde{y}_{l l^{\prime} r k} \leq \theta, & \\
\sum_{l=1}^{S} \sum_{l^{\prime}=1}^{S^{\prime}} \sum_{r=1}^{s} u_{l l^{\prime}}^{\prime} \tilde{y}_{l l^{\prime} r j} \leq C \tilde{I}_{j}^{*} & \forall j, \\
\sum_{l^{\prime}=1}^{S^{\prime}} \sum_{r=1}^{s} u_{l l^{\prime} r}^{\prime}=p_{l} & \forall l, \\
\sum_{r=1}^{s} u_{l l^{\prime} r}^{\prime}=p_{l l^{\prime}}^{\prime} & \forall l, l^{\prime}, \\
u_{l l^{\prime} r}^{\prime}, p_{l l^{\prime}}^{\prime}, p_{l}>0 & \forall l, l^{\prime}, r, \\
\theta \geq 0 . &
\end{array}
$$

The combination of (6)-(12) forms a three-level DEA based-CI model in the log-linear context that identifies the minimum composite loss $\theta_{\min }$ needed to arrive at an optimal set of weights. Constraint (7) ensures that each DMU loses no more than $\theta$ of its best attainable composite value, $C \tilde{I}_{k}^{*}$. The second set of constraints (8) satisfies that the composite values of all DMUs are less than or equal to their upper bound of $C \tilde{I}_{j}^{*}$. Two sets of constraints (9) and (10) are added to the model. This implies that the sum of weights under each (sub-)sub-category equals to the weight of that (sub-)sub-category. It should be noted that the original (or internal) weights used for calculating the weighted averages are obtained as $u_{l l^{\prime} r}=u_{l l^{\prime}}^{\prime} / p_{l l^{\prime}}^{\prime}$ and $p_{l l^{\prime}}=p_{l l^{\prime}}^{\prime} / p_{l}$.

\subsection{Prioritizing Indicator Weights Using AHP}

The three-level DEA based-CI model identifies the minimum composite loss $\theta_{\min }$ needed to arrive at a set of weights of indicators by the internal mechanism of DEA. On the other hand, the priority weights of indicators, and the corresponding (sub-)categories are defined out of the internal mechanism of DEA by AHP.

In order to more clearly demonstrate how AHP is integrated into the three-level DEA-based CI model, this research presents an analytical process in which indicator weights are bounded by the AHP method. The AHP procedure for imposing weight bounds may be broken down into the following steps:

Step 1: A decision maker makes a pairwise comparison matrix of different criteria, denoted by $A$, with the entries of $a_{l q}(l=q=1,2, \cdots, S)$. The comparative importance of criteria is provided by the decision maker using a rating scale. Saaty [7] recommends using a 1-9 scale. 
Step 2: The AHP method obtains the priority weights of criteria by computing the eigenvector of matrix $A$ (Equation (13)), $w=\left(w_{1}, w_{2}, \cdots, w_{S}\right)^{\mathrm{T}}$, which is related to the largest eigenvalue, $\lambda_{\max }$.

$$
A w=\lambda_{\max } w .
$$

To determine whether or not the inconsistency in a comparison matrix is reasonable the random consistency ratio, C.R., can be computed by the following equation:

$$
C . R .=\frac{\lambda_{\max }-N}{(N-1) R . I .}
$$

where R.I. is the average random consistency index and $N$ is the size of a comparison matrix. In a similar way, the priority weights of (sub-)sub-criteria under each (sub-)criterion can be computed. To obtain the weight bounds for indicator weights in the three-level DEA-based CI model, this study aggregates the priority weights of three different levels in AHP as follows:

$$
\bar{u}_{l l^{\prime} r}=w_{l} e_{l l^{\prime}} f_{l l^{\prime} r}, \quad \sum_{l=1}^{S} w_{l}=1, \quad \sum_{l^{\prime}=1}^{S^{\prime}} e_{l l^{\prime}}=1 \text { and } \sum_{r=1}^{s} f_{l l^{\prime} r}=1
$$

where $w_{l}$ is the priority weight of criterion $l(l=1, \cdots, S)$ in AHP and $e_{l^{\prime}}$ is the priority weight of sub-criterion $l^{\prime}\left(l^{\prime}=1,2, \cdots, S^{\prime}\right)$ under criterion $l$ and $f_{l^{\prime} r}$ is sub-sub-criterion $r(r=1, \cdots, s)$ under sub-criterion $l^{\prime}$.

In order to estimate the maximum composite loss $\theta_{\max }$ necessary to achieve the priority weights of indicators for each DMU the following set of constraints is added to the three-level DEA-based CI model:

$$
u_{l l^{\prime} r}^{\prime}=\alpha \bar{u}_{l l^{\prime} r} \quad \forall l, l^{\prime}, r \text {, while } \alpha>0 \text {. }
$$

The set of constraints (16) changes the AHP computed weights to weights for the new system by means of a scaling factor $\alpha$. The scaling factor $\alpha$ is added to avoid the possibility of contradicting constraints leading to infeasibility or underestimating the relative composite scores of DMUs [8].

\subsection{Parametric Goal Programming Model}

In this stage we develop a parametric goal programming model that can be solved repeatedly to generate the various sets of weights for the discrete values of the parameter $\theta$, such that $\theta_{\min } \leq \theta \leq \theta_{\max }$. The purpose of the model is to minimize the total deviations from the priority weights of indicators with a city block distance measure. Choosing such a distance measure, each deviation is being equally weighted subject to the following constraints:

$$
\begin{array}{ll}
\operatorname{Min} Z_{k}(\theta)=\sum_{l=1}^{S} \sum_{l^{\prime}=1}^{S^{\prime}} \sum_{r=1}^{s}\left(d_{l l^{\prime} r}^{+}+d_{l l^{\prime} r}^{-}\right) \\
\text {s.t. } \\
\begin{array}{ll}
u_{l l^{\prime} r}^{\prime}-d_{l l^{\prime} r}^{+}+d_{l l^{\prime} r}^{-}=\alpha \bar{u}_{l l^{\prime} r} & \forall l, l^{\prime}, r, \\
d_{l l^{\prime} r}^{+}, d_{l l^{\prime} r}^{-} \geq 0 & \forall l, l^{\prime}, r,
\end{array}
\end{array}
$$

and constraints (7)-(12).

Here, $d_{l^{\prime} r}^{+}$and $d_{l^{\prime} r}^{-}$are the positive and negative deviations from the priority weight of indicator $r$ under sub-category l' of category l, for $\mathrm{DMU}_{k}$. The set of equations (18) indicates the goal equations whose right-hand sides are the priority weights of hierarchical indicators adjusted by the obtained value of the scaling variable in (16).Because the range of deviations computed by the objective function is different for each DMU, it is necessary to normalize it by using relative deviations rather than absolute ones. Hence, the normalized deviations can be computed by:

$$
\Delta_{k}(\theta)=\frac{Z_{k}^{*}\left(\theta_{\min }\right)-Z_{k}^{*}(\theta)}{Z_{k}^{*}\left(\theta_{\min }\right)},
$$

where $Z_{k}^{*}(\theta)$ is the optimal value of objective function (17) for $\theta_{\min } \leq \theta \leq \theta_{\max }$. We define $\Delta_{k}(\theta)$ as a measure of closeness which represents the relative closeness of each DMU to the weights obtained from the three- 
level DEA based-CI model in the range $[0,1]$ after adding the set of constraints (16) to it. Increasing the parameter $(\theta)$, we improve the deviations between the two systems of weights obtained from the three-level DEA based-CI model before and after adding the set of constraints (16). This may lead to different ranking positions for each DMU in comparison to the other DMUs. It should be noted that in a special case where the parameter $\theta=\theta_{\max }=0$, we assume $\Delta_{k}(\theta)=1$.

\section{Numerical Example}

In this section we present the application of the proposed approach to assess the road safety performance of a set of European countries (or DMUs). The data for eight hierarchical indicators that compose road safety performance indicators (SPIs) for 11 European countries has been adopted from [9]. Table 1 presents the normalized data, using (1) and (2), for SPIs on a logarithmic scale.

The notations in Table 1 are as follows: $\tilde{y}_{1}=$ Road user behavior, $\tilde{y}_{2}=$ Vehicle, $\tilde{y}_{11}=$ Alcohol, $\tilde{y}_{12}=$ Seat belt, $\quad \tilde{y}_{111}=$ Roadside police alcohol tests per 1000 population in 2008, $\tilde{y}_{112}=$ The percentage of drivers above legal alcohol limit in roadside checks in 2008, $\tilde{y}_{121}=$ Daytime seat belt wearing rates on front seats aggregated of cars in 2009, $\tilde{y}_{122}=$ Daytime wearing rates of seat belts on rear seats of cars in 2009, $\tilde{y}_{21}=\tilde{y}_{211}=$ The average percentage of occupant protection score for new cars sold in 2008, $\tilde{y}_{22}=\tilde{y}_{221}=$ The average percentageof pedestrian protection score for new cars sold in 2008, $\tilde{y}_{23}=\tilde{y}_{231}=$ Renewal rate of passenger cars in 2007, $\tilde{y}_{24}=\tilde{y}_{241}=$ Median age of passenger cars in 2008. $\mathrm{AT}=$ Austria, $\mathrm{BE}=$ Belgium, $\mathrm{BG}=$ Bulgaria, $\mathrm{CY}=$ Cyprus, $\mathrm{CZ}=$ Czech Republic, DK = Denmark, EE = Estonia, FI = Finland, FR = France, DE = Germany, EL = Greece.

The results of the AHP model for prioritizing hierarchical SPIs as constructed by the author in Expert Choice software are presented in Table 2. One can argue that the priority weights of SPIs must be judged by road safety experts. However, since the aim of this section is just to show the application of the proposed approach on numerical data, we see no problem to use our judgment alone.

Solving the three-level DEA based-CI model for the country under assessment, we obtain an optimal set of weights with minimum composite loss $\left(\theta_{\min }\right)$. It should be noted that the composite value of all countries calculated from the three-level DEA based-CI model is identical to that calculated from the one-level DEA based-CI model. Therefore, the minimum composite loss for the country under assessment is $\theta_{\min }=0$ (Table 3). This implies that the measure of relative closeness to the AHP weights for the country under assessment is $\Delta_{k}\left(\theta_{\min }\right)=0$. On the other hand, solving the three-level DEA based-CI model for the country under assessment

Table 1. Normalized data for hierarchical SPIs on a logarithmic scale.

\begin{tabular}{|c|c|c|c|c|c|c|c|c|}
\hline \multirow{3}{*}{ Country } & \multicolumn{4}{|c|}{$\tilde{y}_{1}$} & \multicolumn{4}{|c|}{$\tilde{y}_{2}$} \\
\hline & \multicolumn{2}{|c|}{$\tilde{y}_{11}$} & \multicolumn{2}{|c|}{$\tilde{y}_{12}$} & \multirow{2}{*}{$\begin{array}{l}\tilde{y}_{21} \\
\tilde{y}_{211}\end{array}$} & \multirow{2}{*}{$\begin{array}{l}\tilde{y}_{22} \\
\tilde{y}_{221}\end{array}$} & \multirow{2}{*}{$\begin{array}{l}\tilde{y}_{23} \\
\tilde{y}_{231}\end{array}$} & \multirow{2}{*}{$\begin{array}{l}\tilde{y}_{24} \\
\tilde{y}_{241}\end{array}$} \\
\hline & $\tilde{y}_{111}$ & $\tilde{y}_{112}$ & $\tilde{y}_{121}$ & $\tilde{y}_{122}$ & & & & \\
\hline $\mathrm{AT}$ & 0.579 & 0.523 & 0.517 & 0.505 & 0.558 & 0.412 & 0.448 & 0.558 \\
\hline $\mathrm{BE}$ & 0.555 & 0.553 & 0.382 & 0.469 & 0.587 & 0.313 & 0.698 & 0.570 \\
\hline BG & 0.654 & 0.625 & 0.480 & 0.352 & 0.395 & 0.364 & 0.010 & 0.249 \\
\hline $\mathrm{CY}$ & 0.430 & 0.519 & 0.382 & 0.010 & 0.698 & 0.698 & 0.641 & 0.435 \\
\hline $\mathrm{CZ}$ & 0.539 & 0.666 & 0.552 & 0.372 & 0.463 & 0.545 & 0.209 & 0.233 \\
\hline DK & 0.650 & 0.423 & 0.603 & 0.557 & 0.496 & 0.503 & 0.517 & 0.558 \\
\hline $\mathrm{EE}$ & 0.567 & 0.688 & 0.517 & 0.487 & 0.587 & 0.503 & 0.375 & 0.233 \\
\hline FI & 0.010 & 0.682 & 0.603 & 0.683 & 0.644 & 0.545 & 0.295 & 0.415 \\
\hline FR & 0.417 & 0.614 & 0.698 & 0.646 & 0.587 & 0.412 & 0.448 & 0.511 \\
\hline $\mathrm{DE}$ & 0.497 & 0.362 & 0.683 & 0.691 & 0.587 & 0.313 & 0.517 & 0.511 \\
\hline EL & 0.506 & 0.621 & 0.272 & 0.039 & 0.463 & 0.503 & 0.448 & 0.375 \\
\hline
\end{tabular}


Table 2. The AHP hierarchical model for SPIs.

\begin{tabular}{cccc}
\hline Objective level & Criteria level & Sub-criteria level & Sub-sub-criteria level \\
\hline & & Alcohol $e_{11}=0.60$ & Roadside police alcohol tests $f_{111}=0.40$ \\
& $\begin{array}{c}\text { Road user behavior } \\
w_{1}=0.65\end{array}$ & Seat belt $e_{12}=0.40$ & Driving above legal alcohol limit $f_{112}=0.60$ \\
& & Seat belt wearing in front seats $f_{121}=0.70$ \\
$\begin{array}{c}\text { Prioritizing road } \\
\text { safety performance } \\
\text { indicators }\end{array}$ & Vehicle $w_{2}=0.35$ & Pedestrian protection for cars $e_{22}=0.20$ & Pedestrian protection for cars $f_{221}=1.00$ \\
& & Renewal rate of passenger cars $e_{23}=0.40$ & Renewal rate of passenger cars $f_{231}=1.00$ \\
& & Age of passengercars $e_{24}=0.10$ & Age of passenger cars, $f_{241}=1.00$ \\
\hline
\end{tabular}

Table 3. Minimum and maximum losses in composite values for each country.

\begin{tabular}{cccc}
\hline Countries & $C \tilde{I}_{k}^{*}$ & $\theta_{\min }$ & $\theta_{\max }$ \\
\hline AT & 1.000 & 0.000 & 0.1516 \\
BE & 1.000 & 0.000 & 0.1594 \\
BG & 1.000 & 0.000 & 0.3328 \\
CY & 1.000 & 0.000 & 0.1942 \\
CZ & 1.000 & 0.000 & 0.1626 \\
DK & 1.000 & 0.000 & 0.1621 \\
EE & 1.000 & 0.000 & 0.0462 \\
FI & 1.000 & 0.000 & 0.1142 \\
FR & 1.000 & 0.000 & 0 \\
DE & 1.000 & 0.000 & 0.2289 \\
EL & 1.000 & 0.000 & 0.3466 \\
\hline
\end{tabular}

after adding the set of constraints (16), we adjust the priority weights of hierarchical SPIs obtained from AHP in such a way that they become compatible with the weights' structure in the three level DEA-based CI model. Table 4 presents the optimal weights of hierarchical SPIs as well as its scaling factor for all countries. It should be noted that the priority weights of AHP used for incorporating weight bounds on indicator weights after adding (16) to the three-level model are obtained as $\bar{u}_{l l^{\prime} r}=\frac{u_{l l^{\prime} r}^{\prime}}{\alpha}$. Similarly, the priority weights of AHP at criteria level can be obtained as $w_{l}=\frac{p_{l}}{\alpha}$ while $\sum_{l^{\prime}=1}^{S^{\prime}} \sum_{r=1}^{s} u_{l l^{\prime} r}^{\prime}=p_{l}$ and $\sum_{r=1}^{s} u_{l l^{\prime} r}^{\prime}=p_{l l^{\prime}}^{\prime}$.

In addition, the priority weights of AHP at sub-criteria and sub-sub-criteria levels can be obtained as $e_{l l^{\prime}}=p_{l l^{\prime}}^{\prime} / p_{l}$ and $f_{l l^{\prime} r}=u_{l l^{\prime} r}^{\prime} / p_{l l^{\prime}}^{\prime}$, respectively.

The maximum composite loss for each country to achieve the corresponding weights in the three-level DEA-based CI model after adding (16) is equal to $\theta_{\max }$ (Table 3). As a result, the measure of relative closeness to the priority weights of SPIs for the country under assessment is $\Delta_{k}\left(\theta_{\max }\right)=1$.

Going one step further to the solution process of the parametric goal programming model, we proceed to the estimation of total deviations from the AHP weights for each country while the parameter $\theta$ is $0 \leq \theta \leq \theta_{\max }$. Table 5 represents the ranking position of each country based on the minimum deviation from the priority weights of indicators for $\theta=0$. It should be noted that in a special case where the parameter $\theta=\theta_{\max }=0$ we 
Table 4. Optimal weights of hierarchical SPIs obtained from three-level DEA based-CI model bounded by AHP weights for all countries.

\begin{tabular}{ccc}
\hline Weights of categories & Weights of sub-categories & $\begin{array}{c}\text { Weights of } \\
\text { sub-sub-categories }\end{array}$ \\
\hline$p_{1}=1.2322$ & $p_{11}^{\prime}=0.7393$ & $u_{111}^{\prime}=0.2957$ \\
& $u_{112}^{\prime}=0.4436$ \\
& $p_{12}^{\prime}=0.4929$ & $u_{121}^{\prime}=0.3450$ \\
& $p_{21}^{\prime}=1.991$ & $u_{122}^{\prime}=0.1479$ \\
$p_{2}=0.6636$ & $p_{22}^{\prime}=0.1327$ & $u_{211}^{\prime}=0.1991$ \\
& $p_{23}^{\prime}=0.2654$ & $u_{221}^{\prime}=0.1327$ \\
& $p_{24}^{\prime}=0.0664$ & $u_{231}^{\prime}=0.2654$ \\
$\alpha=1.8957$ & & $u_{241}^{\prime}=0.0664$ \\
\hline
\end{tabular}

Table 5. The ranking position of each country based on the minimum distance to priority weights of hierarchical SPIs.

\begin{tabular}{ccc}
\hline Countries & $Z^{*}\left(\theta_{\min }\right)$ & Rank \\
\hline AT & 0.9288 & 9 \\
BE & 0.4619 & 5 \\
BG & 1.2375 & 10 \\
CY & 0.3956 & 4 \\
CZ & 0.8410 & 8 \\
DK & 0.5135 & 6 \\
EE & 0.1307 & 2 \\
FI & 0.3775 & 3 \\
FR & 0.0000 & 1 \\
DE & 0.7205 & 7 \\
EL & 1.5300 & 11 \\
\hline
\end{tabular}

assume $\Delta_{k}(\theta)=1$. Table 5 shows that France (FR) is the best performer in terms of the $C \tilde{I}$ value and the relative closeness to the priority weights of indicators in comparison to the other countries. Nevertheless, increasing the value of $\theta$ from 0 to $\theta_{\max }$ has two main effects on the performance of the other countries: improving the degree of deviations and reducing the value of composite indicator. This, of course, is a phenomenon, one expects to observe frequently. The graph of $\Delta(\theta)$ versus $\theta$, as shown in Figure 1 , is used to describe the relation between the relative closeness to the priority weights of indicators and composite loss for each country. This may result in different ranking positions for each country in comparison to the other countries (Table A1).

\section{Conclusion}

We develop a multiplicative (or log-linear) aggregation approach based on DEA and AHP methodologies to construct CIs for hierarchical indicators. We define two sets of weights of hierarchical indicators in a threelevel DEA framework. All indicators are treated as benefit type which satisfy the property of "the larger the better". The first set represents the weights of indicators with minimum composite loss. The second set represents 


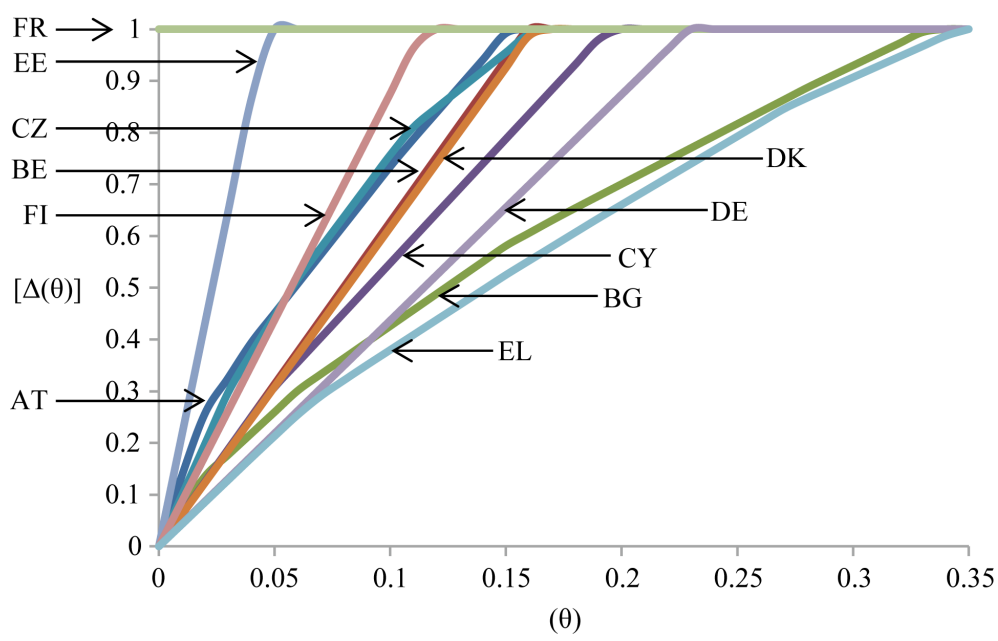

Figure 1. The relative closeness to the priority weights of hierarchical indicators $[\Delta(\theta)]$, versus composite loss $(\theta)$ for each country.

the corresponding priority weights of hierarchical indicators, using AHP, with maximum composite loss. We assess the performance of each DMU in comparison to the other DMUs based on the relative closeness of the first set of weights to the second set of weights. Improving the measure of relative closeness in a defined range of composite loss, we explore the various ranking positions for the DMU under assessment in comparison to the other DMUs. To demonstrate the effectiveness of the proposed approach, we apply it to construct a composite road safety performance index for eight hierarchical indicators that compose SPIs for 11 European countries.

\section{References}

[1] Pakkar, M.S. (2014) Using Data Envelopment Analysis and Analytic Hierarchy Process to Construct Composite Indicators. Journal of Applied Operational Research, 6, 174-187.

[2] Zhou, P., Ang, B.W. and Zhou, D.Q. (2010) Weighting and Aggregation in Composite Indicator Construction: A Multiplicative Optimization Approach. Social Indicators Research, 96, 169-181. http://dx.doi.org/10.1007/s11205-009-9472-3

[3] Shen, Y., Hermans, E., Brijs, T. and Wets, G. (2013) Data Envelopment Analysis for Composite Indicators: A Multiple Layer Model. Social Indicators Research, 114, 739-756. http://dx.doi.org/10.1007/s11205-012-0171-0

[4] OECD (2008) Handbook on Constructing Composite Indicators: Methodology and User Guide. OECD Publishing.

[5] Charnes, A., Cooper, W.W., Seiford, L. and Stutz, J. (1982) A Multiplicative Model for Efficiency Analysis. SocioEconomic Planning Sciences, 16, 223-224. http://dx.doi.org/10.1016/0038-0121(82)90029-5

[6] Hashimoto, A. and Wu, D.A. (2004) A DEA-Compromise Programming Model for Comprehensive Ranking. Journal of the Operation Research Society of Japan, 47, 73-81.

[7] Saaty, T.S. (1980) The Analytic Hierarchy Process. McGraw-Hill, New York.

[8] Podinovski, V.V. (2004) Suitability and Redundancy of Non-Homogeneous Weight Restrictions for Measuring the Relative Efficiency in DEA. European Journal of Operational Research, 154, 380-395. http://dx.doi.org/10.1016/S0377-2217(03)00176-0

[9] Bax, C., Wesemann, P., Gitelman, V., Shen, Y., Goldenbeld, C., Hermans, E., Doveh, E., Hakkert, S., Wegman, F. and Aarts, L. (2012) Developing a Road Safety Index. Deliverable 4.9 of the EC FP7 Project DaCoTA. 


\section{Appendix}

Table A1. The measure of relative closeness to the priority weights of hierarchical SPIs $\left[\Delta_{k}(\theta)\right]$ vs. composite loss $[\theta]$ for each country.

\begin{tabular}{|c|c|c|c|c|c|c|c|c|c|c|c|}
\hline$\theta$ & $\mathrm{AT}$ & $\mathrm{BE}$ & BG & CY & $\mathrm{CZ}$ & DK & $\mathrm{EE}$ & FI & FR & $\mathrm{DE}$ & EL \\
\hline 0 & 0.0000 & 0.0000 & 0.0000 & 0.0000 & 0.0000 & 0.0000 & 0.0000 & 0.0000 & 1.0000 & 0.0000 & 0.0000 \\
\hline Rank & N/A & N/A & N/A & N/A & N/A & N/A & N/A & N/A & 1 & N/A & N/A \\
\hline 0.01 & 0.1373 & 0.0627 & 0.0695 & 0.0627 & 0.0993 & 0.0617 & 0.2163 & 0.0875 & 1.0000 & 0.0437 & 0.0428 \\
\hline Rank & 3 & 7 & 6 & 8 & 4 & 9 & 2 & 5 & 1 & 10 & 11 \\
\hline 0.02 & 0.2576 & 0.1255 & 0.1358 & 0.1253 & 0.1987 & 0.1234 & 0.4326 & 0.1751 & 1.0000 & 0.0874 & 0.0856 \\
\hline Rank & 3 & 7 & 6 & 8 & 4 & 9 & 2 & 5 & 1 & 10 & 11 \\
\hline 0.03 & 0.3261 & 0.1882 & 0.1771 & 0.1880 & 0.2980 & 0.1850 & 0.6489 & 0.2626 & 1.0000 & 0.1311 & 0.1283 \\
\hline Rank & 3 & 6 & 9 & 7 & 4 & 8 & 2 & 5 & 1 & 10 & 11 \\
\hline 0.04 & 0.3942 & 0.2510 & 0.2184 & 0.2506 & 0.3742 & 0.2467 & 0.8652 & 0.3502 & 1.0000 & 0.1747 & 0.1711 \\
\hline Rank & 3 & 6 & 9 & 7 & 4 & 8 & 2 & 5 & 1 & 10 & 11 \\
\hline 0.05 & 0.4508 & 0.3137 & 0.2597 & 0.3076 & 0.4454 & 0.3084 & 1.0000 & 0.4377 & 1.0000 & 0.2184 & 0.2131 \\
\hline Rank & 3 & 6 & 9 & 8 & 4 & 7 & 1 & 5 & 1 & 10 & 11 \\
\hline 0.06 & 0.5075 & 0.3765 & 0.3009 & 0.3556 & 0.5166 & 0.3701 & 1.0000 & 0.5253 & 1.0000 & 0.2621 & 0.2542 \\
\hline Rank & 5 & 6 & 9 & 8 & 4 & 7 & 1 & 3 & 1 & 10 & 11 \\
\hline 0.07 & 0.5642 & 0.4392 & 0.3329 & 0.4036 & 0.5775 & 0.4318 & 1.0000 & 0.6128 & 1.0000 & 0.3058 & 0.2906 \\
\hline Rank & 5 & 6 & 9 & 8 & 4 & 7 & 1 & 3 & 1 & 10 & 11 \\
\hline 0.08 & 0.6209 & 0.5020 & 0.3639 & 0.4516 & 0.6373 & 0.4935 & 1.0000 & 0.7004 & 1.0000 & 0.3495 & 0.3212 \\
\hline Rank & 5 & 6 & 9 & 8 & 4 & 7 & 1 & 3 & 1 & 10 & 11 \\
\hline 0.09 & 0.6776 & 0.5647 & 0.3950 & 0.4997 & 0.6970 & 0.5551 & 1.0000 & 0.7879 & 1.0000 & 0.3932 & 0.3509 \\
\hline Rank & 5 & 6 & 9 & 8 & 4 & 7 & 1 & 3 & 1 & 10 & 11 \\
\hline 0.1 & 0.7342 & 0.6275 & 0.4260 & 0.5477 & 0.7568 & 0.6168 & 1.0000 & 0.8755 & 1.0000 & 0.4368 & 0.3799 \\
\hline Rank & 5 & 6 & 10 & 8 & 4 & 7 & 1 & 3 & 1 & 9 & 11 \\
\hline 0.11 & 0.7874 & 0.6902 & 0.4571 & 0.5957 & 0.8103 & 0.6785 & 1.0000 & 0.9630 & 1.0000 & 0.4805 & 0.4089 \\
\hline Rank & 5 & 6 & 10 & 8 & 4 & 7 & 1 & 3 & 1 & 9 & 11 \\
\hline 0.12 & 0.8385 & 0.7530 & 0.4881 & 0.6437 & 0.8477 & 0.7402 & 1.0000 & 1.0000 & 1.0000 & 0.5242 & 0.4379 \\
\hline Rank & 5 & 6 & 10 & 8 & 4 & 7 & 1 & 1 & 1 & 9 & 11 \\
\hline 0.13 & 0.8897 & 0.8157 & 0.5191 & 0.6917 & 0.8835 & 0.8019 & 1.0000 & 1.0000 & 1.0000 & 0.5679 & 0.4669 \\
\hline Rank & 4 & 6 & 10 & 8 & 5 & 7 & 1 & 1 & 1 & 9 & 11 \\
\hline 0.14 & 0.9408 & 0.8785 & 0.5502 & 0.7397 & 0.9192 & 0.8635 & 1.0000 & 1.0000 & 1.0000 & 0.6116 & 0.4959 \\
\hline Rank & 4 & 6 & 10 & 8 & 5 & 7 & 1 & 1 & 1 & 9 & 11 \\
\hline 0.15 & 0.9919 & 0.9412 & 0.5811 & 0.7877 & 0.9550 & 0.9252 & 1.0000 & 1.0000 & 1.0000 & 0.6553 & 0.5249 \\
\hline Rank & 4 & 6 & 10 & 8 & 5 & 7 & 1 & 1 & 1 & 9 & 11 \\
\hline 0.16 & 1.0000 & 1.0000 & 0.6058 & 0.8357 & 0.9908 & 0.9869 & 1.0000 & 1.0000 & 1.0000 & 0.6990 & 0.5524 \\
\hline Rank & 1 & 1 & 10 & 8 & 6 & 7 & 1 & 1 & 1 & 9 & 11 \\
\hline 0.17 & 1.0000 & 1.0000 & 0.6305 & 0.8837 & 1.0000 & 1.0000 & 1.0000 & 1.0000 & 1.0000 & 0.7426 & 0.5797 \\
\hline Rank & 1 & 1 & 10 & 8 & 1 & 1 & 1 & 1 & 1 & 9 & 11 \\
\hline
\end{tabular}




\begin{tabular}{|c|c|c|c|c|c|c|c|c|c|c|c|}
\hline \multicolumn{12}{|c|}{ Continued } \\
\hline 0.18 & 1.0000 & 1.0000 & 0.6542 & 0.9317 & 1.0000 & 1.0000 & 1.0000 & 1.0000 & 1.0000 & 0.7863 & 0.6071 \\
\hline Rank & 1 & 1 & 10 & 8 & 1 & 1 & 1 & 1 & 1 & 9 & 11 \\
\hline 0.19 & 1.0000 & 1.0000 & 0.6774 & 0.9798 & 1.0000 & 1.0000 & 1.0000 & 1.0000 & 1.0000 & 0.8300 & 0.6343 \\
\hline Rank & 1 & 1 & 10 & 8 & 1 & 1 & 1 & 1 & 1 & 9 & 11 \\
\hline 0.2 & 1.0000 & 1.0000 & 0.7006 & 1.0000 & 1.0000 & 1.0000 & 1.0000 & 1.0000 & 1.0000 & 0.8737 & 0.6607 \\
\hline Rank & 1 & 1 & 10 & 1 & 1 & 1 & 1 & 1 & 1 & 9 & 11 \\
\hline 0.21 & 1.0000 & 1.0000 & 0.7238 & 1.0000 & 1.0000 & 1.0000 & 1.0000 & 1.0000 & 1.0000 & 0.9174 & 0.6871 \\
\hline Rank & 1 & 1 & 10 & 1 & 1 & 1 & 1 & 1 & 1 & 9 & 11 \\
\hline 0.22 & 1.0000 & 1.0000 & 0.7470 & 1.0000 & 1.0000 & 1.0000 & 1.0000 & 1.0000 & 1.0000 & 0.9611 & 0.7135 \\
\hline Rank & 1 & 1 & 10 & 1 & 1 & 1 & 1 & 1 & 1 & 9 & 11 \\
\hline 0.23 & 1.0000 & 1.0000 & 0.7703 & 1.0000 & 1.0000 & 1.0000 & 1.0000 & 1.0000 & 1.0000 & 1.0000 & 0.7398 \\
\hline Rank & 1 & 1 & 10 & 1 & 1 & 1 & 1 & 1 & 1 & 1 & 11 \\
\hline 0.24 & 1.0000 & 1.0000 & 0.7935 & 1.0000 & 1.0000 & 1.0000 & 1.0000 & 1.0000 & 1.0000 & 1.0000 & 0.7662 \\
\hline Rank & 1 & 1 & 10 & 1 & 1 & 1 & 1 & 1 & 1 & 1 & 11 \\
\hline 0.25 & 1.0000 & 1.0000 & 0.8167 & 1.0000 & 1.0000 & 1.0000 & 1.0000 & 1.0000 & 1.0000 & 1.0000 & 0.7926 \\
\hline Rank & 1 & 1 & 10 & 1 & 1 & 1 & 1 & 1 & 1 & 1 & 11 \\
\hline 0.26 & 1.0000 & 1.0000 & 0.8399 & 1.0000 & 1.0000 & 1.0000 & 1.0000 & 1.0000 & 1.0000 & 1.0000 & 0.8190 \\
\hline Rank & 1 & 1 & 10 & 1 & 1 & 1 & 1 & 1 & 1 & 1 & 11 \\
\hline 0.27 & 1.0000 & 1.0000 & 0.8631 & 1.0000 & 1.0000 & 1.0000 & 1.0000 & 1.0000 & 1.0000 & 1.0000 & 0.8454 \\
\hline Rank & 1 & 1 & 10 & 1 & 1 & 1 & 1 & 1 & 1 & 1 & 11 \\
\hline 0.28 & 1.0000 & 1.0000 & 0.8864 & 1.0000 & 1.0000 & 1.0000 & 1.0000 & 1.0000 & 1.0000 & 1.0000 & 0.8668 \\
\hline Rank & 1 & 1 & 10 & 1 & 1 & 1 & 1 & 1 & 1 & 1 & 11 \\
\hline 0.29 & 1.0000 & 1.0000 & 0.9081 & 1.0000 & 1.0000 & 1.0000 & 1.0000 & 1.0000 & 1.0000 & 1.0000 & 0.8868 \\
\hline Rank & 1 & 1 & 10 & 1 & 1 & 1 & 1 & 1 & 1 & 1 & 11 \\
\hline 0.3 & 1.0000 & 1.0000 & 0.9296 & 1.0000 & 1.0000 & 1.0000 & 1.0000 & 1.0000 & 1.0000 & 1.0000 & 0.9068 \\
\hline Rank & 1 & 1 & 10 & 1 & 1 & 1 & 1 & 1 & 1 & 1 & 11 \\
\hline 0.31 & 1.0000 & 1.0000 & 0.9510 & 1.0000 & 1.0000 & 1.0000 & 1.0000 & 1.0000 & 1.0000 & 1.0000 & 0.9268 \\
\hline Rank & 1 & 1 & 10 & 1 & 1 & 1 & 1 & 1 & 1 & 1 & 11 \\
\hline 0.32 & 1.0000 & 1.0000 & 0.9725 & 1.0000 & 1.0000 & 1.0000 & 1.0000 & 1.0000 & 1.0000 & 1.0000 & 0.9468 \\
\hline Rank & 1 & 1 & 10 & 1 & 1 & 1 & 1 & 1 & 1 & 1 & 11 \\
\hline 0.33 & 1.0000 & 1.0000 & 0.9939 & 1.0000 & 1.0000 & 1.0000 & 1.0000 & 1.0000 & 1.0000 & 1.0000 & 0.9668 \\
\hline Rank & 1 & 1 & 10 & 1 & 1 & 1 & 1 & 1 & 1 & 1 & 11 \\
\hline 0.34 & 1.0000 & 1.0000 & 1.0000 & 1.0000 & 1.0000 & 1.0000 & 1.0000 & 1.0000 & 1.0000 & 1.0000 & 0.9868 \\
\hline Rank & 1 & 1 & 1 & 1 & 1 & 1 & 1 & 1 & 1 & 1 & 11 \\
\hline 0.35 & 1.0000 & 1.0000 & 1.0000 & 1.0000 & 1.0000 & 1.0000 & 1.0000 & 1.0000 & 1.0000 & 1.0000 & 1.0000 \\
\hline Rank & 1 & 1 & 1 & 1 & 1 & 1 & 1 & 1 & 1 & 1 & 1 \\
\hline
\end{tabular}

